Le Fantasme de Natures: Réinscrire le Sensible dans la Corporalité

Emilie Dionne

Dans son ouvrage Ecology: Key Concepts in Critical Theory, l'auteure Caroline Merchant avait pour objectif de tracer les liens entre la pensée écologiste, son développement et ses courants, ainsi que de la théorie critique de Francfort; ces théoriciens, tout particulièrement Hébert Marcuse, sur lequel nous nous concentrerons dans cette étude, ont proposé une lecture dialectique de l'Histoire où, assujetties à une (dé)raison, ou plutôt à une raison objective devenue rationalité subjective, natures intérieure et extérieure se sont vues dépouillées de leur autonomie. Sans néanmoins s'entendre sur l'origine de cette dialectique, on s'accorde, entre théoriciens critiques et environnementalistes, à penser que l'exploitation humains/natures va de pair.

Dans les lignes qui suivent, nous nous intéresserons au concept de nature, de même qu'à la possibilité d'émancipation chez Marcuse, lequel s'est brièvement penché sur la crise écologique. Devant un discours environnementaliste et écoféministe multiple se revendiquant de notions diverses, soit l'opposition entre nature/culture, désir/besoin, et en replongeant dans le mythe d'une Nature-Mère, nous proposons une réflexion sur le rapport à la corporalité et à la sensibilité chez Marcuse, sa récupération par l'écoféministe Vandana Shiva, de même que sur ce que l'on apprend des identités (pré)politiques au cours de cette discussion sur la nature.

Notre lecture s'effectuera en revisitant les propos de Marcuse sur les natures et sa position quant à l'hétéronomie des sociétés occidentales; puis nous introduirons une réflexion sur le processus d'essentialisation des genres ainsi que sur l'arrachement $d u$ désir en nous référant aux théories écoféministes; enfin, nous adresserons une brève critique autant 
à la théorie critique de Marcuse qu'à la position écologiste de Shiva en proposant quelques pistes de réconciliation, entre natures, sciences, et désirs.

\section{Le Concept de Nature chez Marcuse}

$\mathrm{Au}$ cœur de son ouvrage Counter-Revolution and Revolt, Marcuse publie un chapitre sur la nature, le politique et la révolte, pressentant la portée émancipatoire alliant la libération des natures (extérieure/intérieure) des humains et des non-humains. À cette époque donc, Marcuse était déjà en mesure de percevoir en quoi la maîtrise et les dominations des natures s'étaient imbriquées l'une dans l'autre, mais plus encore - et c'est en cela qu'il diffère de ses confrères Adorno et Horkheimer - il ose voir dans la nature un sujet pour renverser l'ordre hétéronomique des sociétés actuelles. Ainsi, c'est à partir de l'exploitation de la nature que les humains en sont venus à s'exploiter eux-mêmes, à se soumettre aux contraintes d'une exploitation toujours plus efficiente et performative, au détriment de leur liberté naturelle, de leurs désirs et instincts.

Dans l'optique de résumer son idée, signalons que l'auteur convoitait la possibilité d'exalter une sensibilité nouvelle, pouvant être renforcée en dénichant cet agent auparavant peu considéré, soit la Nature. Dès lors, celle-ci devient un sujet, à mesure que la société, les technologies et les sciences la transforment. Conséquement, Marcuse se pose contre une vision conceptualisant la nature tel un objet inerte, pure, prémoderne, en se référant au niveau de première et seconde natures, plutôt qu'à l'inquiétante opposition de nature/culture; dès lors, la nature est, ou plutôt devient, historique, et ce sont les rapports que nous entretenons avec cette dernière qui doivent être réadressés, en vue de dépasser la subjectivisation et l'état d'aliénation présent.

De quelle manière cette nouvelle sensibilité dans les rapports, à laquelle Marcuse nous exulte, prend-elle forme? D'où émerge-t-elle? 
Dans l'optique de mieux la cerner, il importe de retourner à l'ouvrage Éros \& Civilisation, et de se référer aux théories de Freud sur les énergies de l'Éros et du Thanatos, revisitées par Marcuse. Alors que Freud déplorait les impulsions/instincts sexuels et bestiaux, intrinsèques au principe de vie/plaisir (l’Éros), comme étant incompatibles avec la formation civilisationnelle et le progrès, c'est uniquement, dira-t-il, par une sublimation répressive que se crée la civilisation: c'est-à-dire, par l'affaiblissement du principe du plaisir, par son confinement sublimé dans l'art ou libéré mais exclusivement dans les marges de la reproduction sexuelle/procréatrice. Ici, deux sphères séparées et imperméables sont créées (travail/plaisir), la première étant réservée à la production, au progrès, d'où l'impossibilité de laisser le sensible, le plaisir, troubler l'efficience. ${ }^{1}$

Toutefois, Marcuse nous force à considérer la présence d'un second principe, le Thanatos, principe de mort et de destruction. En état d'équilibre, les deux principes autorisent l'existence et la progression de la civilisation humaine; ici, l'auteur, en contradiction avec la thèse freudienne, s'insurge d'un Éros affaibli et soumis à la domination d'un Thanatos, lequel, en conquérant, conduit inévitablement à la destruction de l'humanité, à l'annihilation de la vie. Cette désérotisation du travail et de la civilisation par la répression du principe du plaisir entraîne la désensibilisation des individus, leur aliénation de la société et de la nature, lesquels n'éprouvent plus aucun plaisir au contact d'autrui; l'instinct de mort, en déséquilibre, engendre violence, domination des humains/non-humains, et destruction de la diversité biologique ou humaine. ${ }^{2}$ Ici, son propos porte avant tout sur la canalisation de l'énergie humaine créatrice en une force bénéfique pour les natures; en revalorisant l'apport créatif de l'Éros, ou de l'imagination, de l'intuition, naît l'éventualité d'étendre une énergie sexuelle, actuellement limitée à

\footnotetext{
${ }^{1}$ Marcuse, 1973

${ }^{2}$ Marcuse, 1973
} 
la reproduction, à un désir érotique et sensuel, à l'expérience sensible ainsi qu'à l'enchantement des natures, ou même des rapports entre individus (pouvant être, ajoutons-nous, humains ou non). ${ }^{3}$

Marcuse considérait dès lors que la "libération" des natures résidait dans la revalorisation des désirs, d'un équilibre retrouvé entre Éros et Thanatos, dont la possibilité se réalisera essentiellement par l'expérience sensible, presque sensuelle, d'où la centralité des désirs. Conséquemment, l'auteur s'aventurera même jusqu'à concevoir un principe féminin, lequel sera par la suite récupéré, revu et réadressé par les écoféministes. ${ }^{4}$ Pour Marcuse, l'archétype «femme» a été fabriqué par la société patriarcale ainsi que par la déraison de la rationalité; si cette dernière est considérée comme étant sensible, plus près de sa corporalité, voire même de $s a$ et de la nature, cela est pour l'essentiel une conséquence du développement de la modernité, ayant engendré leur marginalisation des sphères centrales de la vie sociétale. De fait, le patriarcat crée son propre contrepouvoir, non pas, dans une vision marxiste, le prolétariat, mais plutôt le principe féminin. L'auteur soutient donc que les femmes, non pas en tant que femmes, mais plus spécifiquement le principe féminin qui, dû aux actions du capitalisme patriarcal et de la création/renforcement du genre et de la division sexuelle, se matérialise presque exclusivement dans les corps féminins, elles en deviennent les agents révolutionnaires aptes à dissoudre la société patriarcale, le capitalisme et la déraison de la modernité.

Même si Marcuse demeure fortement opposé à une vision téléologique de la libération des natures et s'il tente d'éviter toute forme d'essentialisme au sein de sa vision du principe féminin, les mots prononcés parviennent difficilement à contrer la chose qu'il met en scène, soit que les femmes semblent bien facilement incarner les agentes universelles de l'émancipation, dès lors qu'elles demeurent incapables de

\footnotetext{
${ }^{3}$ Sandilands, 2001

${ }^{4}$ Et par Shiva, entre autre, auteure à laquelle nous reviendrons dans une section ultérieure.
} 
se distancier du modelage, de la figure que le patriarcat capitaliste a fait d'elles-mêmes. Conséquemment, lorsque Marcuse insiste sur le fait que le principe féminin n'est pas la maternité ou issue d'une quelconque qualité naturelle, il n'en renforce pas moins une tendance à l'essentialisation, en embrassant, ou en échouant à se distancier de la fabrication essentialiste des genres par une raison subjective moderne.

\section{Apport de l'Écoféminisme : Le Processus d'essentialisation}

Il peut sembler contradictoire d'initier une conception historique en abordant le concept d'essence, et il nous apparaît nécessaire d'élaborer sur ce que nous entendons par cette tendance à l'essentialisation. Dans l'optique de développer cette idée, nous nous référerons spécifiquement à l'écoféministe Shiva, qui s'interroge sur la manière dont la Rationalité, la Science moderne ainsi que la société patriarcale ont procédé à une essentialisation des genres, mais plus particulièrement des femmes. Tout d'abord, l'auteure refuse une posture biologique des femmes dans tout discours ou action politique. De fait, pour Shiva, c'est précisément le processus scientifique moderne visant à réduire les femmes à certaines fonctions biologiques qui est problématique; la rationalité scientifique moderne, en ce sens, a confiné les femmes à des corps, à des ventres qui doivent être contrôlés, disciplinés, et ce même vocabulaire est progressivement récupéré par nombre de discours environnementaux radicaux portant notamment sur la surpopulation. À la limite, elles sont vues, lorsqu'on ne les considère que sous un aspect réducteur biologique, comme étant incapables de s'ajuster, de contrôler elles-mêmes leurs fonctions biologiques et, dans l'optique de se reproduire de manière raisonnable, elles doivent être réglées par les technologies, les sciences, les médecins, et les gynécologues parce qu'elles ne parviennent pas à le faire individuellement. ${ }^{5}$

\footnotetext{
${ }^{5}$ Heller, 1999
} 
C'est dans cette perception du passé mythique, idéal, quasiment utopique, que se démarquent les élans «essentialistes ${ }^{6}$ de Shiva; le regard qu'elle jette sur la Nature, sur la Femme par excellence, fait du contact maternel, entre la mère (nature) et ses enfants (humains/nonhumains), le lien primordial nous rappelant notre condition naturelle, non seulement parce que, en tant que femmes, elles créent la vie, mais parce qu'elles prennent en charge les conditions de l'émergence de la vie et ce, même si cette tâche s'est forgée historiquement. ${ }^{7} \mathrm{Si}$, à la base, les femmes ne sont pas essentiellement marquées, nous notons un processus d'essentialisation dans les structures même de l'organisation sociale universelle des femmes. En ce sens, pour être femme, pour être pleinement femme, faut-il être mère, selon Shiva, et être une bonne mère, emplie de compassion, d'attention, de douceur, d'oubli de soi?

Maternité, ici - et l'argument n'en est que renforcé - ne signifie pas simplement enfanter mais prend corps dans la conception d'un « instinct maternel », argument qui, historiquement et socialement, a déterminé ce que la femme était/devait être; selon Shiva, la science moderne, précisément, confine les femmes à leur biologie, les réduisant à des machines reproductives ou à des objets sexuels, devant être contrôlées par le marché (biotechnologies) ou par la religion. ${ }^{8}$ Toutefois, être maternelle selon Shiva, non pas au sens de l'enfantement biologique

Nous nous référons ici à l'ouvrage de Heller sur la relation entre l'amour courtois et les discours environnementaux radicaux. Ainsi, valorisant une conception de la bonne Mère, qui engendre mais de manière contrôlée (grâce au travail scientifique), le slogan de Earth First! "Love your Mother (don't become one) » conçoit le désir humain pour la Nature telle une soif insatiable, toujours destructive. En somme, les humains doivent contenir leurs "désirs charnels ", intrinsèquement incontrôlables et exécrables, visant à exploiter les « fruits de la nature »; cette transformation romantique de l'amour, et de la nature, nourrit une conception de la sexualité essentiellement reproductive, sinon perverse. Le corps est dévalué et ses élans doivent être freinés par les idées, la Raison. La figure de Gaïa, ainsi, renforce une sexualité hétérosexuelle reproductive, orientée vers les désirs d'un seul des deux partenaires (dans l'amour romantique et la nature romantisée, l'homme-chevalier). De plus, elle tend à dévaluer les autres mères, les mauvaises mères, les femmes pauvres et sous-éduquées dans les pays du Sud, celles qui n'embrassent pas de fait la rationalité scientifique chevaleresque apte à rétablir le rapport avec La Nature.

${ }^{6}$ Ici, nous employons le concept «essentialisme» pour démontrer que Shiva elle-même ne rompt pas complètement avec les dualismes nature/culture; l'essence qu'elle retire du principe féminin, de la maternité, réduit les femmes à leurs fonctions biologiques et porte une conception figée de l'essence, laquelle est peu dynamique, ou évolutive.

${ }^{7}$ Shiva \& Mies, 1993; Shiva, 1998

Ici, l'expression courante "donner la vie" nous permet de savourer toute la portée d'un sacrifice qui est celui de leur sexe certes, mais aussi de leur condition sociale et communautaire.

${ }^{8}$ Shiva, 2005 
mais dans l'acte de la compassion, perpétue une vision particulière de l'identité femme; ne pas être maternelle serait d'adhérer au patriarcat, soit ne plus/pas être femme. Dès lors, le principe féminin étant synonyme de maternité, rejoint le mythe Mère-Nature, de Gä̈a ${ }^{9}$, c'est peut-être historiquement que les femmes sont devenues les gardiennes des enfants, des communautés, mais Shiva souhaite une extension anhistorique de cette identité, prépolitique, immuable, pour atteindre les objectifs de la soutenabilité entre humains et non-humains. ${ }^{10}$

Même, elle critique ardemment les féministes du Nord de par la séparation que ces dernières valorisent et exultent entre procréation et désir/vitalité sexuelle, en affirmant que les concepts sont indissociables. Évidemment, ce que Shiva cherche à illustrer par cette véhémence implique que par la rupture de l'activité/désir sexuel avec la reproduction, les agissements naturels des individus sont conçus comme incontrôlables et devant être surveillés/ajustés par les technologies; cette obstination à se limiter à une échelle individuelle pour comprendre l'agir humain, plutôt qu'à l'expression de cultures sociales, alimente le mythe selon lequel la révolution scientifique constitue une réussite en matière de compréhension, de contrôle et de dépassement des phénomènes naturels. ${ }^{11}$ Voilà en somme la critique que Shiva adresse aux femmes ayant embrassé la tendance libérale, la biotechnologie, l'oubli de leur

\footnotetext{
${ }^{9}$ Bien que le discours écoféministe, particulièrement celui valorisant la position des femmes dans le rapport à la nature, de même qu'exaltant la position des mères, se veut émancipateur pour la cause des femmes, il n'en tend pas moins, parallèlement, à soulever nombre de questionnements, précisément lorsque cette position peut être confondue avec celle de la consolation, de l'isolement dans la sphère privée, ainsi qu'une essentialisation des genres aux sexes : "If women allow themselves to be consoled for their culturally determined lack of access to the modes of intellectual debate by the invocation of hypothetical great goddesses, they are simply flattering themselves into submission (a technique often used on them by men). All the mythic versions of women, from them myth of redeeming purity of the virgin to that of the healing, reconciling mother, are consolatory nonsenses; and consolatory nonsense seems to me a frail definition of myth, anyway. Mother goddesses are just as silly a notion as father gods. If a revival of the myths of these cults gives woman emotional satisfaction, it does so at the price of obscuring the real conditions of life. This is why they were invented in the first place. Myth deals in false universals, to dull the pain of particular circumstances. In no area is this more true than in that of relations between the sexes. » (Carter, 1978).

${ }^{10}$ Shiva effectue cette association problématique, qui signale que pour être pleinement femme, nous devons être mère. Sous-entendu dans cette association - puisque Shiva aborde le thème de la famille, les relations homme/femme, et l'indissociabilité de la procréation et du désir sexuel - est que pour être pleinement femme, il faut être mère et pour être mère, il faut avoir un enfant, scellant le destin de femme de par sa dépendance envers l'homme (Alaimo, 2000).

${ }^{11}$ Shiva, 2005
} 
corporalité, en sous-entendant que ces femmes aliénées sombrent dans la monstruosité.

Ici, visiblement Marcuse reprocherait à Shiva d'avoir elle-même incorporé le processus civilisationnel selon Freud qu'il transforme luimême, soit que le progrès social implique une désérotisation des corps, lesquels désirs ne sont confinés $q u$ 'à la procréation. Son incapacité à distancier la procréation du désir sexuel pose problème puisqu'ils perpétuent normativement un modèle sexuel, familial axé sur des relations hétérosexuelles. Si elle célèbre le corps/instrument de contact du monde, il est curieux qu'elle n'en valorise pas les désirs sensuels hors d'une sphère exclusivement individuelle/égoïste, qu'elle dissocie sensibilité de sensualité. Cette vision réductrice du désir (sexuel), en se référant ici à, engendre plutôt que conteste la réduction de la sexualité à la reproduction hétérosexuelle et, aussi, à un discours sur les comportements individuels rationnels. ${ }^{12}$

\section{L'Arrachement du Désir}

Réarticulant ce que nous avons abordé précédemment à travers les mots de Marcuse, nous notons que ces femmes (du Nord) sont victimes de l'arrachement de désir, c'est-à-dire de la scission entre travail et sensible, corps et esprit, puisqu'elles doivent nier leur corporalité, dans l'optique d'accomplir pleinement leur insertion dans le système standardisé, capitaliste et rationnel; en termes de rendement, toute sensibilité, curiosité et désir lié à la corporalité est anéanti pour la simple productivité. Ainsi, le corps est un instrument de travail, d'où sa désensibilisation puisque le désir le ralentit, le pousse à explorer, à être séduit; dès lors, la position de Shiva, et de Marcuse, serait de réintroduire

\footnotetext{
${ }^{12}$ Sandilands, 1998

C'est dans cette optique qu'il importe de valoriser une dénaturalisation du désir, toujours en référence à Sandilands, soit sa distanciation d'un discours sur la nature associant exclusivement désir sexuel à l'hétérosexualité et à la reproduction.
} 
le sensuel aux corps, lui rendant sa passion et son rôle d'instrument de connaissance/expérience au monde.

La question du désir, cependant, soulève certaines questions et demeure généralement occultée dans le discours environnemental puisqu'on tend à opposer désir et besoin ${ }^{13}$, et à se revendiquer des besoins pour exposer l'urgence de la tâche environnementale et des désirs, perçus comme fondamentalement égoïstes, destructeurs de la nature. En ce sens, les écoféministes - et Shiva n'y échappe pas désirant introduire davantage la vision des femmes, offriront et même se ressourceront à l'image pure et détachée de ces dernières, à une femmemère qui donne et se sacrifie pour ses enfants (non pas uniquement biologiquement), pour sa communauté. Conséquemment, selon Shiva, ces désirs que la technologie et les sciences justement ont détournés des questions concrètes, communautaires, sont à condamner, puisqu'ils ne chercheraient qu'à combler des besoins superficiels, esthétiques et destructeurs.

Ainsi, plusieurs écoféministes traiteront la question de la technologie, tout particulièrement des technologies de la reproduction, de leur réception et de leur utilisation par les femmes de contextes sociaux et culturels divers. Là où les femmes (issues de pays occidentaux notamment) optent pour la biotechnologie pour faciliter plusieurs aspects de leur vie sociale et professionnelle, et diminuer les douleurs/contraintes relatives à leurs cycles biologiques, d'autres femmes ${ }^{14}$ sont alors victimes des mêmes technologies, stérilisées ou avortées pour éviter de donner naissance à des filles, lesquelles sont dévaluées socialement. Selon Merchant et Ariel Salleh, il ne fait aucun doute que la science moderne a dépossédé les femmes d'un savoir sur la reproduction; désormais, les appareils existent pour compenser aux irrégularités, voire

\footnotetext{
${ }^{13} \mathrm{Si}$ le mouvement environnementaliste romantique fut bien populaire dans les années 60-70, la valorisation de la beauté et de l'esthétisme seule, de nos jours, ne parvient guère à accroître la sensibilisation à la crise écologique, entre autre pour les interrogations de classe intrinsèquement soulevées lorsqu'on oppose "beauté de la nature" à "subvenir aux besoins" des affamés.

${ }^{14}$ Nous nous référons ici précisément aux cas abordés par Shiva, donc au cas des femmes en Inde.
} 
aux faiblesses biologiques/naturelles des femmes. ${ }^{15}$ Dans le but de donner naissance, les femmes sont soumises aux technologies et aux sciences des hommes, munis de forceps, d'étriers, de masques, niant leurs muscles et leurs capacités. ${ }^{16}$

En ce sens-là, la femme traditionnelle aurait moins à désapprendre que la femme technologique ou les hommes, puisqu'elle ne s'est pas encore travestie; elle n'a pas été incorporée dans les systèmes centraux de l'exploitation. La femme du Nord, de fait, est libre uniquement dans les sphères de son aptitude à se comporter tel un homme, à faire disparaitre les contraintes biologiques. Pour Shiva, et Marcuse, le corps se doit plutôt d'être un instrument de conscience, c'est à partir de lui que l'on fait l'expérience du monde. ${ }^{17}$

Jamais neutre puisque créée et produite dans le contexte du patriarcat, cette suspicion envers la science et la technologie moderne nous apparaît problématique; en effet, une évacuation entière des technologies engendre des doutes dans la mesure où il n'est nullement envisagé que celles-ci puissent être réappropriées et réadressées par les groupes en fonction des contextes, perpétuant plutôt une dissociation ontologique entre femme et technologie, tel que suggéré par Marcuse. ${ }^{18}$ S'il est vrai que l'on ne peut extraire les technologies de leur contexte de création et, qu'en ce sens, elles ne seront jamais neutres, il n'en demeure pas moins que leurs rôles n'en sont pas déterminés, contrairement à ce que prône Shiva. Plutôt, la démonisation de la technique/technologie provoquera une spirale de déshumanisation et de déresponsabilisation à mesure que les actions, les décisions et la manipulation qui se terrent derrière ces instruments plongent dans un certain obscurantisme. ${ }^{19}$

\footnotetext{
${ }^{15}$ Merchant, 1980; Salleh 1988

${ }^{16}$ Marcuse, 1973; Horkheimer 1974

${ }^{17}$ Là où Shiva s'opposera à Marcuse, c'est en clamant que la technologie n'est qu'une barrière entre le monde non-humain et la corporalité.

${ }^{18}$ Curtis, 1998

${ }^{19}$ Heller, 1999
} 
En définitive, Shiva a raison de scander que la science moderne n'a pas su apporter le contrôle des natures puisque les femmes prémodernes connaissaient les cycles de leur corps et parvenaient déjà à contrôler et à pratiquer contraception et accouchement. Toutefois, l'incapacité à embrasser une distinction novatrice entre le contrôle disciplinaire, exercé par ceux qui détiennent actuellement les moyens (bio)technologiques, et le sens d'habiter son corps, en employant de manière respectueuse/créative ces savoirs et technologies, limite son propos. ${ }^{20}$

\section{Conclusion: Dépasser les limites d'un désir émancipatoire}

En terminant, si l'apport des penseurs critiques demeure pertinent lorsque nous tentons de réfléchir sur les concepts de natures, et sur les identités politiques, en vue de s'interroger sur la tendance à se référer dans les mouvements sociaux actuels à des identités prépolitiques, on peut néanmoins se demander jusqu'à quel point la possibilité de libération ou d'émancipation mise en scène par Marcuse mais également par Shiva n'en vient pas à incarner l'un des obstacles principaux auquel se heurte la réflexion. De fait, cette option émancipatoire ne nous enferme-t-elle pas, plutôt, dans une forme de ce que Timothy Luke, en référence à Foucault, nommait la 'biogouvernementalité', où le privé, les modes de vie, deviennent des données à surveiller et à contrôler; ici, la notion d'émancipation elle-même semble dès lors plus téléologique que dynamique. Ainsi, peut-on prescrire la libération ou l'émancipation? Ce discours ne contient-il pas déjà une certaine forme d'enfermement, d'aliénation, en ordonnant selon des lignes directrices détenues par certains les actions de tous, une forme d'organisation disciplinaire?

Brièvement, dans cette étude, nous avons vu en quoi la revendication d'un principe féminin demeure hautement suspicieuse et inquiétante, de par le fait qu'elle est incapable de se dissocier entièrement des dualismes qu'elle veut critiquer. Conséquemment, est-il

\footnotetext{
${ }^{20}$ Twine, 2001
} 
possible, voire souhaitable, de constituer un sujet révolutionnaire solide et exclusif? En somme, Marcuse soulève aussi un point essentiel à notre question, qui est malheureusement ignoré par les écoféministes, soit la question des nouvelles manières d'aborder les sciences. En s'extirpant d'une vision dualisme nature/culture ${ }^{21}$, et en embrassant plutôt de nouvelles figures identitaires fluides et dynamiques, il est possible autant de dépasser les nœuds actuels de la pensée critique que dans la pensée environnementale et féministes. Au cœur de mon propos se trouve le renoncement à des formes pures, le désir de réfléchir des formes hybrides, des agents qui modifient et se modifient continuellement et de manière dynamique; en revisitant la relation entre les machines, les métissages et les natures, de même que les sociétés, peut-être parviendrait-on à contester les catégories identitaires problématiques auxquelles se confrontent autant les environnementalistes, les féministes que les écoféministes. L'artificialité, en ce sens, c'est déjà la nature, si on s'attarde à une relecture de Marcuse et à une vision plus généreuse du rapport entre naturalité et artificialité.

\section{$\underline{\text { Références }}$}

Alaimo, A. (2000). Undomesticated Grounds: Recasting Nature as a Feminist Space. Ithaca: Cornell University Press.

Carter, A. (1978). The Sadeian Woman: and the Ideology of Pornography, Bloomsbury : Virago Editions.

Curtis, D.(1997). Women's Knowledge as Expert Knowledge: Indian Women and Ecodevelopment, in Warren, K.J. (Ed.).

\footnotetext{
${ }^{21}$ Il apparaît pertinent de revenir brièvement sur la question de l'essentialisme, lequel est originairement faussé puisque la nature et la culture sont indissociables; ici, visiblement, si le processus est historique, il n'en ait pas moins forcé sur ce que les femmes doivent être, comment elles doivent se comporter, soit embrasser le système et devenir des mères dans le sens total. De fait, l'idée veut donc que biologiquement les potentialités sont certes finies, mais non pas déterminées, et le social, la culture, permettent l'activation de certaines d'entre elles, tout en en laissant d'autres inactives; elles les utilisent, les redéfinissent, les réadressent continuellement. Parler d'une distinction entre nature et culture ne fait guère de sens désormais, alors que l'essence elle-même se construit.
} 
Ecofeminism: Women, Culture, Nature. Bloomington: Indiana University Press.

Heller, C. (1999). Ecology of Everyday Life: Rethinking the desire for nature. Montréal: Black Rose Books.

Horkheimer, M. (1974). Eclipse of Reason. New York: Seabury Press.

Marcuse, H. (2003). Éros \& Civilisation. Paris: Minuit. . (1973). Contre-Révolution et Révolution. Paris: Seuil.

Merchant, C. (1999). Key Concepts in Critical Theory: Ecology. New York: Humanity Books. . (1980). The death of nature: women, ecology, and the scientific revolution. San Francisco: Harper \& Row.

Salleh, A.(1988). Epistemology and the Metaphors of Production: An Eco-Feminist Reading of Critical Theory. Studies in the Humanities. 15(2), pp.130-139.

Sandilands, C. (2001) Desiring Nature, Queering Ethics: Adventures in Erotogenics Environments. Environmental Ethics, 23, pp.169-186.

Sandilands, C. (1998). Sex at the Limits, in Darier, É. (Ed.). Discourses of the Environment. Oxford: Blackwell Publishers.

Shiva, V. (2005). Earth democracy: justice, sustainability, and peace. Cambridge: South End Press. . (1998). Staying alive. London: Zed Books LTD.

Shiva, V. \& Mies, M.(1993). Ecofeminism. Halifax: Fernwood.

Twine, R.T. (2001). Ma(r)king essence-ecofeminism and embodiement. Ethics \& The Environment, 6(2), pp.31-58. 